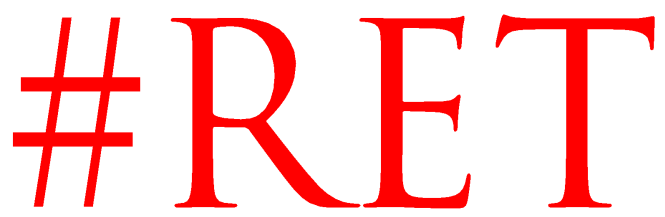

Revista Española de la Transparencia

Núm. 8. Primer Semestre 2019

ISSN 2444-2607. Págs. 101-119

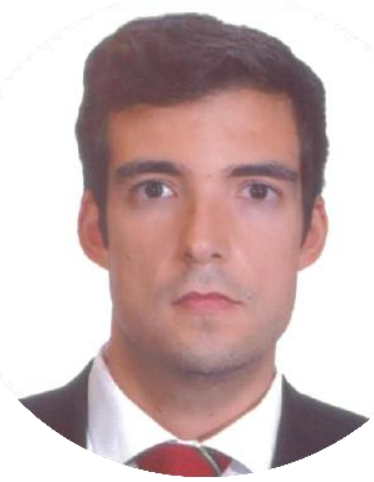

Alfonso Sánchez Garcia1

Universidad de Murcia

\title{
La planificación de los contratos públicos como posible fuente de transparencia administrativa. El caso italiano
}

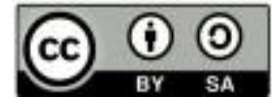

RECIBIDO: 30 de agosto de 2018 ACEPTADO: 31 de enero de 2019

RESUMEN: En el siguiente artículo se analiza la exhaustiva y exigente normativa italiana en materia de programación de contratos públicos a fin de poner de manifiesto como la misma, dada su directa influencia en la validez de los subsiguientes procedimientos de contratación, así como su directa repercusión en la conformación de una información digital a disposición de todos los ciudadanos, contribuirá decididamente a incrementar de manera efectiva la transparencia sobre las decisiones de los entes adjudicadores que culminan por dar lugar a la incoación del correspondiente procedimiento de compra. De esta forma, el Ordenamiento italiano nos muestra un interesante desarrollo normativo destinado a asegurar que todo procedimiento de compra pública responde realmente a un adecuado intento de satisfacer una necesidad directamente vinculada al interés social o a la utilidad pública. Construcción normativa que puede servir de inspiración para un potencial desarrollo en nuestro Ordenamiento Jurídico de la disposición general contenida en el art. 28.4 de la Ley 9/2017.

PALABRAS CLAVE: Transparencia, planificación, contratación pública, TIC.

\footnotetext{
${ }^{1}$ Licenciado en Derecho y Administración de Empresas por la Universidad de Murcia ahora realiza sus estudios de Doctorado en dicha Universidad gracias a la concesión de una beca FPU del Ministerio de Universidades. Recientemente ha llevado a cabo una estancia de larga duración en la Scuola di Specializzazione in Studi sull'Amministrazione Pubblica de la Universidad de Bolonia, gracias a la cual se ha tenido acceso a la documentación necesaria para la realización del presente artículo que forma parte de las investigaciones para la realización de la Tesis.
} 


\section{Alfonso Sánchez García}

CONTENIDOS: 1 . La importancia de las comunicaciones en la fase de preparación del procedimiento de contratación pública. - 2.- Configuración legal de La programación de la actividad de compra pública como sistema de preinformación. 2.1. Contenido de la programación de la contratación pública. 2.2. Modificaciones ulteriores de la programación y posibles excepciones a la misma. 2.3. Prescripciones estatales sobre la priorización de los procedimientos de compra pública en la actividad de programación de la Administración Pública. 2.4. Eficacia y efectos juridicos de la programación de la contratación pública. - 3. Efectos esperados de la planificación de los procedimientos de compra pública como instrumento de transparencia. - Bibliografía.

\section{Public Procurement planning as potential source of administrative transparency. The Italian example.}

ABSTRACT: This article analyzes the exhaustive and demanding Italian planning public procurement regulations in order to show how, given its direct influence on the validity of the subsequent tender procedures, as well as its effects by generating digital information available to all citizens, could contribute decisively to the goal of a real growth of transparency about contracting authorities decisions which lead to public procurement procedure opening. In this way, the Italian Legal Framework shows us an interesting regulatory development aimed at ensuring that any public procurement procedure really responds to an adequate attempt to satisfy a need directly linked to social interest or public utility, giving rise to a regulatory structure that can serve as inspiration in Spanish Legal Framework, mainly to address the article 28.4 of the Spanish Public Procurement Law development.

KEYWORDS: Transparency, planning, public procurement, ITC. 


\section{La planificación de los contratos públicos como posible fuente de transparencia administrativa. El caso italiano}

\section{LA IMPORTANCIA DE LAS COMUNICACIONES EN LA FASE DE PREPARACIÓN DEL PROCEDIMIENTO DE CONTRATACIÓN PÚBLICA}

Las comunicaciones relativas a los procedimientos de compra pública efectuadas en el nivel de preinformación se hallan referidas a las actuaciones de preparación de aquellos, esto es, a una fase del procedimiento de contratación previa a la confección definitiva de los pliegos de contratación y de su puesta en conocimiento respecto de terceros. Como señala Scoca (2008: 94, nota 134) y la profusa doctrina por él citado, no podría afirmarse que estemos ante una fase previa a la propia existencia del procedimiento de contratación, sino ante la fase de "deliberazione di contrarre" consistente en "un cierto y propio acto administrativo dirigido a fijar la normativa de aplicación así como los motivos que justifican la incoación del procedimiento". En efecto, durante esta fase se producirá la evaluación de las necesidades y disponibilidades económicas por parte del ente adjudicador que justificará, posteriormente, la incoación del procedimiento de compra, así como los términos técnicos y económicos que figuren en los pliegos. Por tanto, aquella engloba una serie de actuaciones previas indispensables para la obtención de una actuación del ente adjudicador racional tendente al correcto funcionamiento y eficacia de la importante canalización de fondos públicos intrínseca al aprovisionamiento de bienes, servicios, obras y externalización de gestión de servicios públicos (Sciancalepore, 2017: 44).

En efecto, con estas actuaciones el ente adjudicador procederá, supuestamente, a la construcción de las curvas de ingreso público marginal, coste público marginal y a una previsión de la curva de oferta a la que se enfrentará en relación a cada uno de los tipos de productos $u$ obras existentes en el mercado respecto de los que haya detectado cierta necesidad en cuanto a su utilización o disposición. Actuaciones de las que dependerán los parámetros trazados en los pliegos de contratación y, por ende, el punto de equilibrio que se alcanzará en el mercado de la contratación pública y, consecuentemente, la potencial eficiencia del procedimiento. Fuertes implicaciones de la fase de programación en el ulterior desarrollo del procedimiento que han llevado a la Autorità per la Vigilanza sui Contratti Pubblici di lavori, servizi e forniture ${ }^{2}$-AVCP- a situar, en buena parte de los casos, la razón de las carencias detectadas durante la ejecución del mismo en la forma en que se han desarrollado las actuaciones propias de esta primera fase ${ }^{3}$.

\footnotetext{
${ }^{2}$ Autoridad administrativa independiente existente de manera autónoma hasta el año 2014, cuando fue integrada en el seno de la Autorità Nazionale Anticorruzione -ANAC-.

3 AVCP, documento di consultazione, "Servizi e forniture: programmazione, progettazione ed esecuzione del contratto" en el que se viene a expresar la falta de planificación previa como origen de efectos indeseados "tales como la fragmentación de las contrataciones, el recurso frecuente a prórrogas contractuales ilegítimas, el inicio de procedimientos negociados sin publicidad motivados únicamente por la urgencia de abastecer un determinado producto u obra, la imprecisa definición del objeto del contrato en relación a las especificaciones técnicas y/o a la cantidad, la pérdida del control del gasto".
} 


\section{Alfonso Sánchez García}

así como la aparición en el decreto legislativo -en adelante, d.lgs. - n. 50/20164 de una fuerte regulación de esta en sus arts. 21 a 27, y el reconocimiento expreso en su art. 32.2 de una verdadera y preceptiva actividad administrativa a estos efectos. De este modo, podriamos afirmar que la nueva regulación del Ordenamiento italiano ha superado la tradicional conceptualización de la preparación del contrato como una fase que limita sus efectos jurídicos dentro del "ámbito de la estructura burocrática del ente contratante, sin que la misma pueda suponer efectos de ningún género fuera de dicha estructura" (Mele, 2011: 42 y 43), fundamentalmente a través de las programaciones plurianuales de contratos públicos, por las cuales se viene a exigir una realización de una transparente y rigurosa "determinazione di contrarre".

Por todo ello, como pasamos a analizar en detalle, el hecho de exigir la transparencia de las operaciones efectuadas, o que deberian ser efectuadas, durante esta primera fase que podriamos determinar como estructuralmente interna del ente adjudicador, mediante la publicación obligatoria y con una determinada anticipación de las contrataciones futuras que desean ser efectuadas, unido ello a la existencia de mandatos imperativos relativos a su formulación que conectan con su posterior ejecución, supondrá instituir, realmente, una obligación de cumplir fielmente con las actuaciones propias y esperables de dicha fase inicial, en la que al encontrarnos con unos agentes económicos, como son los entes adjudicadores, caracterizados por una fuerte disociación entre los responsables de la contratación y los "accionistas" proveedores de los fondos y beneficiarios de los resultados de la misma (Manchlup, 1979; Monchón, 1979: 163 a 183), se puede detectar una potencial tendencia o favorecimiento estructural a que no se realicen con el debido rigor -o directamente no se realicen- las actividades de planificación y preparación de los procedimientos de contratación. Al mismo tiempo que se permite el establecimiento de un importante foco sobre una fase en la que se desarrollará, inevitable y naturalmente, buena parte de la actividad discrecional del ente adjudicador, permitiendo su "monitorización" (Clarich, 2016: 301).

\section{CONFIGURACIÓN LEGAL DE LA PROGRAMACIÓN DE LA ACTIVIDAD DE COMPRA PÚBLICA COMO SISTEMA DE PREINFORMACIÓN}

El principal tipo de las comunicaciones de "información digital" previstas en la normativa italiana durante la fase de preinformación lo encontramos regulado en el art. 21 del d.lgs. n. 50/2016, por el que se viene a establecer la "necesaria" realización de un "programa bienal de adquisición de bienes y servicios" que alcancen o sobrepasen los cuarenta mil euros y de "otro de carácter trienal para la adquisición de obras" que alcancen o sobrepasen los cien mil euros 5 , así como las

\footnotetext{
${ }^{4}$ Decreto Legislativo 18 aprile 2016, n. 50. Codice dei contratti pubblici, por el que se ha procedido a la trasposición de las directivas de cuarta generación.

${ }^{5}$ Como señala la Corte Costituzionale, Sentenza n. 485/1995 la exigencia de la programación de las obras públicas consiste en la determinación de las obras que pueden ser efectivamente y
} 


\section{La planificación de los contratos públicos como posible fuente de transparencia administrativa. El caso italiano}

obras complejas que probablemente se lleven a cabo mediante contratos de concesión o de colaboración público privada, debiendo procederse, respecto de ambas planificaciones, a su actualización anual. Tales previsiones del art. 21 del d.lgs. n. 50/2016 se hallan limitadas a las "administraciones adjudicadoras", lo que equivaldría en nuestra normativa de contratos al ámbito subjetivo de las Administraciones Públicas.

Estas programaciones deberán confeccionarse "en el respeto de los documentos de programación y en coherencia con el presupuesto" - art. 21.1 del d.lgs. n. 50/2016- lo que entendemos que supone un planteamiento más acertado que el sostenido por el d.lgs. n. 163/20066, el cual preveía su realización de manera conjunta a los presupuestos del ente adjudicador, toda vez que permitirá el desarrollo de aquella a la vista de los recursos disponibles para el respaldo de las actuaciones de aprovisionamiento en los mercados de compra pública.

Además, dicha regulación viene a suponer una importante novedad normativa respecto del art. 128 del d.lgs. n. 163/2006 en el que se limitaban sus previsiones al programa trienal de obras públicas 7 . No obstante, no podemos concluir que el art. 21 del d.lgs. n. 50/2016 suponga la introducción ex novo del programa bienal de compra de bienes y servicios, sino, en todo caso, un "endurecimiento" de su regulación. En efecto, este programa bienal ya era previsto en el art. 271.1 del decreto del Presidente de la República -en adelante, d.P.R. - n. 207/20108 con carácter facultativo ${ }^{9}$ y periodicidad anual, tras lo cual se daría un paso hacia la regulación actual mediante la Legge n. 208/201510 la cual, en el epígrafe 505 de su art. 1 vino a adelantar la preceptividad de tal programación bianual a los contratos de bienes y servicios de más de un millón de euros.

completamente realizadas sobre la base de las disposiciones financieras y de las prioridades existentes derivadas de la valoración de sus coste-beneficio, lo que podemos enlazar con la definición de programación establecida por Giannini (1977: 292) como "la realización de un diseño ordenado de actuaciones proyectadas en el futuro".

${ }^{6}$ Decreto Legislativo 12 aprile 2006, n. 163. Codice dei contratti pubblici relativi a lavori, servizi e forniture in attuazione delle direttive 2004/17/CE e 2004/18/CE.

7 D.lgs. n. 163/2006 que supuso en su día un importante avance respecto de las preocupaciones respecto de la fase de programación, normada positivamente por primera vez por el legislador ex art. 14 de la Legge n. 109/1994 y su desarrollo contenido en los arts. 11 a 14 de su reglamento de desarrollo el d.P.R. n. 554/1999.

8 Decreto del Presidente della Repubblica 5 ottobre 2010, n. 207. Regolamento di esecuzione ed attuazione del decreto legislativo 12 aprile 2006, n. 163.

9 No obstante, como determina la ANAC, Determinazione n. 5/2013, 6 novembre: Linee guida su programmazione, progettazione ed esecuzione del contratto nei servizi e nelle forniture, cit.: "una vez adoptada la programación anual, los servicios y los suministros no recogidos en aquella o no incluidos en las excepciones del epígrafe 5 , segundo periodo, no pueden recibir financiación alguna por parte de las administraciones públicas"

${ }^{10}$ Legge 28 dicembre 2015, n. 208. Disposizioni per la formazione del bilancio annuale e pluriennale dello Stato. Legge di stabilità 2016. 


\section{Alfonso Sánchez García}

\subsection{Contenido de la programación de la contratación pública.}

Las programaciones de contratos de obras y de contratos de bienes y servicios deberán contener la previsión de todos y cada uno de estos contratos que se pretendan llevar a cabo, siendo ya detallado el contenido de ambos en el decreto del Ministero delle Infrastrutture n. 77323/2014 y, previamente, en el Decreto n. 59082/2011, a lo que se unieron las determinaciones al respecto llevadas a cabo por la AVCP ${ }^{11}$.

Actualmente, el contenido de las programaciones se halla regulado por las disposiciones del Decreto n. 14/2018 ${ }^{12}$. En concreto, su artículo 6.2, en relación con su Anexo II, viene a exigir que las programaciones bianuales de bienes y servicios incluyan los siguientes extremos:

- La identificación de los recursos necesarios para la realización de las compras previstas en el programa desglosadas por anualidad y fuente de financiación.

- Y la identificación de cada una de las compras programadas. Empleando para ello el llamado Codice Unico di Intervento - CUI- que se asignará en función de su primera inserción en el programa de planificación -art. 2.1, lett. d) del decreto en cuestión-, el Codice Unico di Progetto -CUP_- establecido por el art. 11 de la Legge n. 3/2003 para la identificación de cada proyecto de inversión pública, el codice fiscale de la Administración contratante -el cual vendría a ser el equivalente a nuestro $\mathrm{CIF}$-, la región o regiones en las que se llevará a cabo la ejecución de la compra, el código CPV, una descripción mediante texto de la compra y si se trata de un suministro o un servicio.

A dichos elementos de identificación-clasificación se deberá añadir información adicional consistente en: la primera anualidad en la que el proyecto de compra programado haya sido introducido; la anualidad en la que se pretende dar inicio a la contratación; si se trata o no de una compra integrada dentro del importe de otro procedimiento de contratación, incluida en su correspondiente programación bianual o trienal, así como el CUI del mismo si se trata de un lote funcional según la definición contenida en el art. 3.1, let. qq) del d.lgs. n. 50/2016; su nivel de prioridad;

\footnotetext{
${ }^{11}$ AVCP, Determinazione n. 5 del 6 novembre 2013. Linee guida su programmazione, progettazione ed esecuzione del contratto nei servizi e nelle forniture.

12 El art. 9 del referido decreto señala que la regulación expuesta entrará en vigor a partir de la conformación de las programaciones trienales de obras públicas correspondientes al periodo 20192021 y a las programaciones bienales de bienes y servicios correspondientes al periodo 2019-2020. Por otro lado, en cuanto a su ámbito de aplicación hay que destacar que, tal y como señala el Consiglio di Stato, Parere n. 351/2017, 13 febbraio, su Decreto MIT relativo alla programmazione triennale e biennale, punto 8 , y en atención a la llamada y autorización llevada a cabo por el propio d.lgs. n. 50/2016 en la redacción original del epígrafe 8 de su art. 21, nos encontramos ante un reglamento de desarrollo que adquirirá el mismo ámbito de aplicación que la norma desarrollada. Dicho decreto viene a derogar y sustituir el desarrollo efectuado en materia de programación de obras públicas llevado a cabo por el decreto del Ministero delle Infrastrutture n. 77323/2014 de 24 de octubre.
} 


\section{La planificación de los contratos públicos como posible fuente de transparencia administrativa. El caso italiano}

el responsable del procedimiento - RUP_; la duración del contrato; si se trata de una nueva partida relativa a un contrato en activo; el desglose de la estimación del gasto en cada uno de los dos años y, en su caso, en años sucesivos, así como su importe total y la aportación, en su caso, de capitales privados; la identificación, en su caso, de la central de compras o sujeto agregador al que se recurrirá para la realización de la correspondiente licitación; así como se si se trata de un elemento introducido ex variación de la programación por causa normativamente prevista.

En cuanto al contenido de la programación trienal de obras -art. 3.2 del decreto-. esta deberá individuar las obras que se iniciarán en la siguiente anualidad, indicando los medios financieros con los que se cubrirá su realización especificados por anualidad y fuentes de financiación. En el caso de las obras de importe superior al millón de euros, se deberá aprobar de manera anticipada el proyecto de factibilidad técnica y económica.

Las obras programadas serán identificadas por el código CUI, por el CUP, y por el código interno utilizado por el ente adjudicador. Igualmente, se emplearán los códigos ISTAT y NUTS. Con ello se conseguirá codificar la información de las obras programadas relativa al lugar de ejecución de la obra, tipología y sector y subsector de la obra, a lo que se unirá una descripción mediante texto de la obra. Si bien, es más que destacable, en este punto, el hecho de que se haya decidido prescindir de la identificación mediante código CPV de las obras programadas, al contrario de lo previsto en relación a las programaciones bianuales de bienes y servicios, lo que ha de merecer nuestra consideración negativa.

Adicionalmente, para cada una de las obras se indicará información adicional consistente en la anualidad en la que se prevé el inicio del procedimiento de licitación correspondiente; el responsable único del procedimiento -RUP-; si constituirá o no un lote funcional; si se trata o no de una obra compleja a la vista de la definición contenida en el art. 3.1, let. oo) del d.lgs. n. 50/2016; el nivel de prioridad de la obra; el importe total estimado de gasto, así como el desglose del mismo por cada uno de los tres primeros años y sucesivos; la aportación de capitales privados; el vencimiento de los capitales obtenidos mediante financiación externa para la realización de la obra; y el valor de los bienes inmuebles disponibles por parte del ente adjudicador que hayan sido transferidos para la realización del objeto de la obra, así como si la obra en cuestión responde a una modificación sobrevenida de la programación.

Indicado lo anterior, para cada una de las concretas intervenciones contenidas en las actuaciones de obra programadas se especificará su finalidad ${ }^{13}$; si existe o no conformidad urbanística; si se ha llevado a cabo la verificación de las obligaciones

\footnotetext{
13 Finalidad clasificable en alguna de las siguientes finalidades: adecuación normativa, calidad ambiental, realización de obra incompleta, conservación de patrimonio, mejora o incremento de servicios, calidad urbana, revalorización de bienes vinculados, demolición de obra incompleta o demolición de obra preexistente cuyo uso ha sido abandonado.
} 


\section{Alfonso Sánchez García}

ambientales; el nivel en el que se encuentre su proyectación; y la identificación de la central de compras a la que, en su caso, se vaya a confiar su licitación -Tablas D y E del Anexo I del decreto-

Con carácter innovador, el art. 21.2 del d.lgs. n. 50/2016 establece la obligación de reconocer en sede de los planes de programación trienales las obras que, por cualquier motivo, una vez iniciadas hayan quedado incompletas, expresión estas, en última instancia, "de un uso poco eficiente de los recursos públicos, así como de la imposibilidad de satisfacer la necesidad de la colectividad a la cual estaba destinada"14. Debiendo valorarse, respecto de tales obras incompletas, si, según las circunstancias de cada caso, existen las condiciones para su culminación, o bien, la posibilidad de su reutilización y destino a uso alternativo, de su cesión como medio de pago para la realización de otra obra pública, de su venta, o, finalmente, tan solo de su demolición. Extremo que ha sido desarrollado en sede del art. 4 y en el Anexo I, Tabla B del decreto n. 14/2018.

Por otro lado, deberán de relacionarse los bienes inmuebles disponibles que pueden ser objeto de cesión, los que presenten un derecho de uso que haya sido cedido con carácter instrumental y conexión técnica respecto de trabajos asignados por concesión -art. 21.5 del d.lgs. n. 50/2016-, así como aquellos que se encuentren disponibles por inexistencia del interés público tras completar una obra pública, resultado representativo de la actuación irreflexiva y poco planificada de las Administraciones Públicas en materia de contratación pública -art. 3.2 en relación al Anexo I, Tabla C del decreto n. 14/2018-.

Finalmente, tanto en el caso de la programación trienal de obras públicas, como en la programación bienal de bienes y servicios, deberá informarse acerca de los procedimientos de compra de bienes y servicios y de las obras que hubieran sido anteriormente programados y que desaparezcan de programaciones ulteriores sin que se haya llevado a cabo el correspondiente procedimiento de licitación, debiendo especificarse el motivo de ello -art. 7.3 y Anexo I, Tabla F del decreto n. 14/2018-.

En el caso en el que se no se tenga intención de proceder a la realización de ninguna obra pública o compra de bienes y servicios, tal y como establecen los arts. 5.8 y 7.4 del decreto, ello deberá ponerse de manifiesto en el apartado de "Administración Transparente" en la sede electrónica, así como en los portales electrónicos normalmente habilitados para la publicación de las programaciones efectuadas.

14 Consiglio di Stato, Parere n. 351/2017, 13 febbraio, su Decreto MIT relativo alla programmazione triennale e biennale, cit 


\section{La planificación de los contratos públicos como posible fuente de transparencia administrativa. El caso italiano}

\subsection{Modificaciones ulteriores de la programación y posibles excepciones a la misma.}

La programación tanto de adquisición de bienes y servicios, como de obras, presenta un sistema de flexibilización de su carácter preceptivo ante determinados supuestos.

El primero de tales instrumentos, recogido en los arts. 5.6 y 7.6 del decreto n. 14/2018, consiste en la posibilidad de actualización o reformulación de la programación en cuestión dentro de los noventa días siguientes desde la entrada en vigor de la Legge di Bilancio -equivalente a la Ley de Presupuestos Generales del Estado- para el caso de los entes estatales y desde la aprobación del correspondiente presupuesto o documento análogo en el resto de Administraciones Públicas.

El segundo de dichos sistemas de flexibilización vendrá constituido por la posibilidad de proceder a la modificación de la programación a lo largo del año corriente, siempre y cuando el objetivo de la modificación se realice a fin de -arts. 5.9 y 7.8 del decreto n. $14 / 2018-{ }^{15}$.

- Cancelar una o más obras o compras de bienes y servicios ya previstas en el listado anual.

- Agregar una o más obras o compras de bienes y servicios tendente a la adecuación a lo dispuesto en un acto administrativo adoptado a nivel estatal o regional.

- Agregar una o más obras o compras por el incremento sobrevenido de las disponibilidades financieras del ente adjudicador no previsible al momento de elaboración de la programación, incluidos aquellos incrementos que se produzcan por rebajas en los precios de adquisición previstos en los procedimientos de licitación previamente ejecutados.

- Anticipar la realización de obras o compras programadas para anualidades posteriores.

\footnotetext{
${ }^{15}$ Tal disposición viene a restringir la libre modificación de la programación permitida por el Decreto del Ministero delle Infrastrutture n. 77323/2014 de 24 de octubre, lo que obligará a la matización de la jurisprudencia anterior recogida, entre otras, en Tribunal Administrativo Regional -T.A.R.- Sicilia. Catania. Sezione I, Sentenza n. 1625/2016, 16 giugno, por la que se establece que podrá procederse a su variación sin necesidad de motivación particular alguna. En efecto, la regulación del nuevo decreto de 2018 si obligará a la motivación para la inclusión sobrevenida en la programación y listado anual de obras -o bienes y suministros en el caso de la programación bianual- de modo que se justifique su inclusión dentro de alguna de las causas habilitantes. No obstante, la cancelación de obras programadas, dada la amplitud con la se contempla su posibilidad dentro del decreto y la inexistencia de un deber de motivación expresamente contemplado, como en el caso del art. 5.7 del decreto, podría mantener la solución juridica dada por la referida Sentencia, así como en lo referente a las actualizaciones de la programación que se lleven a cabo.
} 


\section{Alfonso Sánchez García}

- Modificar el cuadro económico de financiación incluido en la programación, al detectarse la necesidad de recursos adicionales.

Finalmente, los arts. 3.13, 5.11, 6.11 y 7.9 del decreto $n .14 / 2018^{16}$ permiten que se lleven a cabo con total libertad y con independencia de la programación aquellas obras cuya necesidad derive del acaecimiento de eventos imprevisibles o catastróficos $^{17}$ o por el establecimiento sobrevenido de disposiciones legales o reglamentarias.

\subsection{Prescripciones estatales sobre la priorización de los procedimientos de compra pública en la actividad de programación de la Administración Pública.}

A la hora de ejecutar las programaciones trienales de obras públicas y bianuales de bienes y servicios deberá respetarse el orden de prioridad contenido en el mismo, el cual deberá quedar estructurado en función de tres categorías de prioridad posibles: mínima, media y máxima ${ }^{18}$.

A este respecto, el decreto n. 14/2018 viene a establecer que, en todo caso, deberán ser determinados como prioritarios los contratos derivados de eventos catastróficos, el aprovisionamiento de bienes y servicios relativos a necesidades primarias, la finalización de obras incompletas, la realización de obras de manutención, la recuperación del patrimonio existente, la realización de obras con proyectos definitivos o ejecutivos ya aprobados, la realización de obras o adquisición de bienes y servicios cofinanciadas con fondos europeos, así como aquellos casos en los que sea posible la realización de la obra o la adquisición del bien o servicio con posibilidad de financiación mayoritaria mediante capitales privados, y la realización de pedidos destinados a completar compras de bienes y servicios incompletas -arts. 3.11 y 6.10 del decreto-. Por su parte, los procedimientos de compra pública cuya necesidad venga motivada por el acaecimiento de actos administrativos adoptados a nivel estatal o regional podrán, a elección de la Administración contratante, ser previstos con cualquier nivel de prioridad, incluidos los niveles superiores -arts. 3.13 y $6.11-$.

\footnotetext{
${ }^{16}$ Que vienen a suceder las disposiciones previamente contenidas en el art. 128.5 del d.lgs. n. 163/2006, a excepción de lo relativo a procedimientos de compra que hundan su motivación en actos administrativos de carácter nacional o regional, cuya inserción necesitará, como hemos visto, del correspondiente procedimiento de modificación de la programación, aunque con una especial flexibilidad a la hora de determinar su prioridad.

${ }^{17}$ Vemos como la normativa de programación recoge las disposiciones más generales en materia de contratación pública tendentes a la simplificación del procedimiento y a la presteza en la selección del operador adjudicatario en caso de situación catastrófica tal y como señala y desarrolla Dugato (2017: 490 a 494) pudiendo destacar como el autor señala que, incluso en estas situaciones, podría ser recomendable orientar la actuación rápida y de emergencia con la realización de una programación acelerada abierta al debate público que permita replantear la situación y la "re-construcción" de la estructura urbana de cara al futuro, previendo entre otras cosas, posibles soluciones o paliativos ante repeticiones de la catástrofe en cuestión.
}

${ }^{18}$ Anexo I, Tabla D.3 y Anexo II, Tabla B.1 del decreto n. 14/2018. 


\section{La planificación de los contratos públicos como posible fuente de transparencia administrativa. El caso italiano}

Dentro de tales casos prioritarios, deberán ser de un grado de prioridad mayor a los anteriores la terminación de obras incompletas, y de un grado aún mayor la relativa a la realización de obras cuya necesidad derive de catástrofes naturales -art. 3.12 del decreto-, lo que, en relación a la programación trienal de obras públicas, nos conduce a una nueva subdivisión de prioridades dentro de la propia categoría de prioridad superior de los tipos de contratos relacionados en el art. 3.11, la cual quedaría subdividida en una prioridad superior-mínima -las señaladas en el art. 3.11-. superior-media -las obras de finalización de obras incompletas- y superior-máxima -las obras derivadas de catástrofes naturales-.

Ello viene a suponer la determinación normativa, no ya de la obligación de planificación estratégica de la acción de compra pública y de su correspondiente publicación, sino la propia orientación en determinados aspectos de la misma. Orientación respecto de la que podría surgir la duda acerca de si el tenor de los arts. 3.11 y 6.10 del decreto establecen una categoría de prioridad exclusiva para los procedimientos de compra que se encuentren en alguno de los casos anteriormente citados o, sencillamente, la obligación de su categorización con la máxima prioridad, sin perjuicio de que dicha categoría sea empleada para otro tipo de contratos.

En este punto, debe destacarse que el tenor de los preceptos en cuestión, en relación con sus correspondientes anexos, tras establecer que los entes adjudicadores procedan a la elaboración de la programación con la valoración de cada una de las obras programadas como prioridad máxima, media o mínima, determinan que, en todo caso, aquellos "establecerán como prioritarios" los contratos que se encuentren en alguno de los casos enumerados. Ello nos conduce a una expresión literal por la que se exige no ya que se sitúe a los contratos comprendidos en estas categorias dentro de una determinada prioridad, sino que se les establezca en una categoría superior o "prioritaria" a la de todos los demás. De este modo, al establecer la catalogación imperativa de su prioridad de manera comparativa, en el que caso en el que se incluyera una categoría de contratación diversa a las detalladas en los arts. 3.11 y 6.10 en el mismo orden de prioridad o superior al de estas, se estaría incumpliendo el tenor de los referidos preceptos.

Expuesto lo anterior, no podemos dejar de poner de manifiesto las dudas constitucionales, al menos respecto del ámbito de aplicación otorgado a estas disposiciones por al art. 2.2 del d.lgs. n. 50/2016, que surgen en relación a estas previsiones, las cuales podrian entenderse como una merma a la autonomía de los órganos de contratación integrados en un ámbito territorial regional o local, sin el respaldo de las necesarias competencias estatales. Debe recordarse en este punto que el tenor del art. 117.2, lett. g) de la Costituzione limita la competencia del Estado en materia de organización a los entes estatales y nacionales, desprendiéndose consiguientemente una competencia plena de las regiones en materia de organización regional y local por aplicación de la cláusula residual del art. 117.4 de 


\section{Alfonso Sánchez García}

la Costituzione (Ponti, 2005: 130). En consecuencia, y dado que la contratación pública no constituye un ámbito competencial individuado y propio dentro del reparto contenido en el referido art. 117, o una competencia en sentido "técnico" siguiendo la nomenclatura de la Corte Costituzionale, sino un conjunto heterogéneo de competencias de carácter general "de diverso anclaje constitucional (Caringella y Giustiniani, 2015: 44) que podrán abarcar o estar presentes en las materias "técnicas" expresamente contenidas en dicho artículo, siempre con respeto a los principios de proporcionalidad y adecuación (Tassone, 2016: 107)19; a la hora de abordar dicha cuestión deberá procederse al análisis individuado de cada uno de los motivos que conducen al establecimiento de un sistema imperativo de prioridad superior que deberán respetar todos los entes adjudicadores, a fin de analizar su posible encuadramiento en alguna de las competencias exclusivas del Estado o de establecimiento de los principios fundamentales - o normas básicasen competencias compartidas con las regiones.

En primer lugar, el imperativo de ejecución prioritaria por parte de todos los entes adjudicadores de realizar procedimientos de licitación necesarios como consecuencia de una catástrofe natural o para garantizar la satisfacción de intereses públicos primarios podría encontrar su justificación competencial en el art. 117.2 let. h) de la Costituzione, esto es, sobre las competencias del Estado en materia de orden público y seguridad.

De otro lado, en relación a la realización prioritaria de forma imperativa consistente en completar obras incompletas o bien en finalizar adquisiciones de bienes y servicios incompletas, obras con proyectos definitivos o ejecutivos ya aprobados, y obras financiadas mayoritariamente con capital privado o con fondos de la Unión Europea, podría tratar de buscarse su amparo constitucional en el art. 117.2 let. e) de la Costituzione en lo relativo a la competencia del Estado en materia de armonización de los presupuestos públicos, o bien en su competencia de regulación básica en la materia compartida con las regiones de coordinación de las finanzas públicas - art. 117.3 de la Costituzione-, en el sentido de que, cuando en la redacción de los programas de compra pública se detecte por cualquier ente adjudicador la necesidad de actuar en cualquiera de los casos antedichos, se deba dar prioridad a la actuación en tales aspectos con el fin - de solventar situaciones de clara y manifiesta ineficiencia económica del sistema de contratación pública como vienen a representar procedimientos de licitación incoados que no han sido ejecutados según lo previsto; - de ejecutar acciones de obra pública con un horizonte más claro a la hora de su rápida y eficaz ejecución y consiguiente alcance de la fase de obtención de retornos o satisfacción del interés público, como son aquellas obras con proyectos definitivos o ejecutivos ya aprobados; - y de favorecer acciones claramente facilitadoras del equilibrio del presupuesto de la administración contratante, como pueden serlo las obras financiadas

19 Sobre este punto véanse las Senteze de la Corte Costituzionale n. 272/2004, 27 luglio; n. 401/2007. 23 novembre; n. 303/2003, 1 ottobre; n. 44/2014, 13 marzo. 


\section{La planificación de los contratos públicos como posible fuente de transparencia administrativa. El caso italiano}

mayoritariamente con capital de terceras fuentes de financiación tales como el capital privado o los fondos de la UE. Misma línea en la que podrian encuadrarse las obras de manutención y obras de recuperación de patrimonio existente, dado que su realización será necesaria para la consecución de que los activos de los entes adjudicadores se encuentren al cien por cien de sus capacidades a la hora de producir sus correspondientes retornos y amortización, bien en forma de caudal económico, bien en forma de servicio público directo o indirecto ${ }^{20}$. Adicionalmente, este último caso, podría encontrar su fundamento constitucional, ahora desde la perspectiva del administrado, sobre la base del art. 117.2, let. $\mathrm{m}$ ) de la Costituzione y de la competencia básica del Estado en materia de mejora del patrimonio cultural ex art. 117.3.

A todo ello debemos unir el hecho de que la detección -en muchos casos subjetiva- de la necesidad de actuar dentro de alguna de estas materias correrá por cuenta del ente adjudicador, siendo solo a partir del momento de la determinación de la necesidad de actuación en tal sentido cuando actuará la competencia exclusiva del Estado o reguladora de los principios básicos de las materia compartidas, orientando tal actuación en el sentido de conducir imperativamente a su establecimiento en un nivel de prioridad superior. En consecuencia, no estamos ante el establecimiento de una obligación de actuar impuesta por el Estado que modifique la autonomía de los entes adjudicadores, sino tan solo ante una orientación de las decisiones de licitación llevadas a cabo y originadas por iniciativa exclusiva de estos, en cuanto las mismas sean tangenciales con ámbitos de competencia del Estado.

\subsection{Eficacia y efectos jurídicos de la programación de la contratación pública.}

Como se ha tenido ocasión de exponer, la redacción de la programación viene a suponer, en última instancia, un acto interno de la administración, lo que, por lo general, nos llevaría a un ámbito donde las actuaciones del ente adjudicador no podrian ser directamente atacadas por los administrados ${ }^{21}$, pudiendo considerarse que, aunque pasen a ser puestos en conocimiento de terceros, serán insusceptibles de provocar situaciones subjetivas respecto de terceros jurídicamente tutelables, tal y como se determinó en su día respecto de la

\footnotetext{
${ }^{20}$ Conexión entre la normativa sobre contratos y sus efectos esperados similar a la analizada y valorada positivamente en Corte Costituzionale, Sentenza n. 36/2004, 26 gennaio.

${ }^{21}$ En esta fase podría considerarse de aplicación lo señalado en relación a la fase de determinación de contratar, respecto de la que hemos de predicar, con carácter general la "no inmediata impugnabilidad" y su carácter endoprocedimental, tal y como señalan Caringella y Giustiniani (2015: 364) en sintonía con lo expresado por el T.A.R. Campania. Napoli. Sezione I, Sentenza n. 1160/2012, 7 marzo, toda vez que, como indica Galli (2015: 218), p. 218, la fase de programación precedería incluso a la fase de determinación de contratar, alejándose, aún más si cabe, de los primeros actos de exteriorización e iniciación del concreto procedimiento de contratación.
} 


\section{Alfonso Sánchez García}

regulación de la deliberación para contratar contenida en el art. 56 de la Legge n. 142/1990 22 (Mele, 2011: 47).

Sin embargo, la especial trascendencia de la programación en el ulterior desarrollo de los contratos ha venido a consentir la posibilidad de impugnarla directamente, aunque de manera limitada respecto de la primera y original introducción de un contrato y no así respecto de los contratos que traigan causa de programaciones anteriores ${ }^{23}$, al considerarse que la aprobación de la programación no es realmente una actividad meramente interna, sino un acto fundamental de determinación de los objetivos concretos a alcanzar por los entes adjudicadores, el cual podrá ser fiscalizado por los administrados en cuanto a su congruencia y legalidad ${ }^{24}$. Es más, en la impugnación de las programaciones efectuadas por las Administraciones contratantes podriamos encontrar la peculiaridad de que estén legitimados para impugnar, no solo los operadores económicos partícipes en el ulterior procedimiento de contratación, sino cualquier ciudadano, dada la extensión y evidencia objetiva de la contravención del interés público, en este caso, positivado por el decreto n. 14/2018, así como por el hecho de que nos podriamos encontrar ante una de las excepciones establecidas por el Consiglio di Stato a la hora de limitar la legitimidad para impugnar el procedimiento de contratación, en concreto, aquella relativa a la impugnación directa de la opción del ente adjudicador de iniciar el procedimiento ${ }^{25}$.

Adicionalmente, ha de destacarse el hecho de que la mayor transparencia derivada de la publicación preceptiva del contenido de la fase de deliberación de contratar, junto con la existencia de mandatos imperativos que deben ser respetados en su formulación y efectiva ejecución, facilitará que los incumplimientos del procedimiento acaecidos durante dicha fase puedan ser traídos a colación en el seno del concreto procedimiento de contratación tratando de instar su anulación.

Lo anterior vendría a dar respuesta a las posibilidades de actuación de los operadores económicos y ciudadanos en general ante la realización de una priorización de los contratos programados que no se ajuste a las pautas normativas expuestas en el epígrafe anterior ${ }^{26}$, así como a una fiscalización por parte de aquellos de la ejecución de la correspondiente programación (Caminiti, 2017: 375). En relación a esta fiscalización debemos resaltar el caso en el que se pretenda llevar a cabo un procedimiento de contratación no programado, el cual era respondido expresamente por el art. 128.10 del d.lgs. n. 163/2006 al determinar que

\footnotetext{
${ }^{22}$ Legge 8 giugno 1990, n. 142. Ordinamento delle autonomie locali.

${ }^{23}$ T.A.R. Lombardia. Sezione III, Sentenza n. 315/2010, 9 febbraio.

${ }^{24}$ Consiglio di Stato. Sezione V. Sentenza n. 5824/2002, 23 ottobre.

${ }^{25}$ Consiglio di Stato, Adunanza Plenaria, n. 4/2011, 7 aprile.

${ }^{26}$ Sobre el carácter cogente de dichas prioridades véase Consiglio di Stato. Sezione IV. Sentenza n. 651/2016, 18 febbraio.
} 


\section{La planificación de los contratos públicos como posible fuente de transparencia administrativa. El caso italiano}

tales procedimientos no podrian recibir ningún tipo de financiación procedente de una Administración Pública. Dicha previsión expresa ha desaparecido en el ámbito del d.lgs. n. 50/2016, sin embargo, hemos de entender que el testigo de lo previsto en el d.lgs. n. 163/2006 ha sido recogido por la interacción de los arts. 21.1, 21.3, 21.6 y $32.1^{27}$ del d.lgs. n. 50/2016 y el art. 21 -octies de la Legge n. $241 / 1990^{28}$, por los cuales se viene a establecer que, en todo caso, con independencia de la procedencia de la financiación -y al contrario que en el régimen de 2006- será anulable todo procedimiento de contratación que no se haya efectuado de acuerdo a lo previsto en la normativa reguladora de la programación de la contratación pública y, por ende, de todo procedimiento de licitación que no haya sido previamente programado o incluido sobrevenidamente en la correspondiente programación en los casos previstos, salvo que se trate de necesidades derivadas de situaciones imprevisibles, catastróficas o necesarias en virtud de modificaciones legales o reglamentarias. Lo que igualmente deberá entenderse de aplicación respecto de los procedimientos de compra que incumplan las divisiones o subdivisiones de prioridad pertinentes ${ }^{29}$.

Vemos, pues, como la programación de la contratación pública cobra una influencia trascendental sobre la licitud y corrección de los procedimientos de compra pública ulteriores, al tiempo - en buena medida gracias a ello- que aporta una información y una transparencia ciertamente potente. En consecuencia, dicha importancia será heredada por la actuación del RUP, cuya designación ya se prevé en el art. 31 del d.lgs. n. 50/2016 para la fase de programación, siendo preciso asegurar una decidida profesionalización de estos responsables que permita asegurar un alto nivel técnico de las planificaciones de compra y sirva de garantía a

\footnotetext{
${ }^{27}$ Por el que se establece el mandato inequívoco de que los contratos públicos se lleven a cabo "en el respeto de los actos de programación del ente adjudicador previstos en el presente código o en la normativa vigente".

${ }^{28}$ Legge 7 agosto 1990, n. 241. Nuove norme in materia di procedimento amministrativo e di diritto di accesso ai documenti amministrativi.

${ }^{29}$ En cierto modo, en contraposición a la jurisprudencia antes citada y en línea con el criterio general propio de una fase ulterior a la de programación como es la de determinación de contratar, podría tratar de considerarse como extrapolable al caso que ahora nos ocupa la jurisprudencia contenida entre otras en T.A.R. Puglia. Bari. Sezione I, Sentenza n. 725/2000, 1 febbraio, en relación al anuncio de información previa, por la que se viene a sostener que este, en cuanto no constituye iniciación alguna del procedimiento de contratación propiamente dicho, no es susceptible por sí mismo de crear un daño directo, inmediato y concreto a los operadores económicos interesados en el procedimiento. A lo que se une el hecho de que, aun siendo cierto que, al contrario del contenido de los anuncios de información previa, el de las programaciones plurianuales condicionará la ejecución de los procedimientos de contratación pública ulteriores, tal vinculación no llegará a obligar al cumplimiento indefectible de lo programado dada la amplia facultad de modificación en cualquier momento para cancelar las acciones de contratación programadas, tal y como hemos tenido ocasión de analizar. No obstante, al margen de dichas posibilidades de variación, los efectos jurídicos de las programaciones serán notablemente superiores a los de los anuncios de información previa tanto por la posibilidad de su impugnación directa ya referida, como por una configuración normativa que conducirá a que un procedimiento de licitación que se aparte de aquella estará vulnerando indefectiblemente la normativa de aplicación, deviniendo un procedimiento irregular.
} 


\section{Alfonso Sánchez García}

la hora de favorecer que las mismas se orienten, exclusivamente, a la consecución del interés público (Mattarella, 2016: 340 y 341; Dugato, 2015: 672).

\section{EFECTOS DE LA PLANIFICACIÓN DE LOS PROCEDIMIENTOS DE COMPRA PÚBLICA COMO INSTRUMENTO DE TRANSPARENCIA}

La actividad de programación analizada está destinada a procurar, en desarrollo del criterio directivo previsto en la Legge n. 11/201630 en su art. 1.1, let. bb), que el procedimiento de compra pública alcance mayores niveles cualitativos y cuantitativos en la prosecución del interés público, en primer lugar mediante la instauración preceptiva de "una fase importante del asi llamado ciclo de vida del contrato"31 orientada a perfilar la estrategia de aprovisionamiento, la optimización del uso de los recursos y el control de su gestión a fin de contribuir a la consecución del principio de buon andamento, economicidad y eficiencia de la acción administrativa (Caringella y Giustiniani, 2015: 347 y 348; Galli y otros, 2015: 214; Caminiti, 2017: 355 y 356; Comba, 2014: 38 a 41)32, tendente, entre otras cosas, a la eliminación de una dinámica en el funcionamiento de las Administraciones Públicas que origine la toma decisiones cortoplacistas "de última hora" poco eficientes, al tiempo que se garantiza la posibilidad de su cobertura financiera (Caringella y Protto, 2017: 95).

Por otro lado, la información o preinformación facilitada por las programaciones de los procedimientos de contratación pública es lo suficientemente completa como para transmitir las necesidades del poder adjudicador en un determinado mercado de contratación pública, dirigiéndose así, a los operadores económicos que lo componen, con la finalidad de que aquellos que pudieran estar interesados en participar en los subsiguientes procedimientos de contratación pública lleven a cabo las actividades de preparación comercial que les sean necesarias, con la consiguiente obtención de ventajas relativas a la concurrencia de operadores económicos en los futuros procedimientos de contratación.

Y, por otro, se promueven ventajas relativas a la consecución de una conducta tendencialmente más "recta" de los responsables de la contratación que evite la toma de decisiones que puedan responder a intereses particulares en lugar de a reales intereses públicos. Ventajas derivadas de una mayor transparencia de las necesidades del ente adjudicador y de las labores de preparación y diseño de los

\footnotetext{
30 Legge 28 gennaio 2016, n.11. Deleghe al Governo per l'attuazione delle direttive 2014/23/UE, 2014/24/UE e 2014/25/UE.

${ }^{31}$ Consiglio di Stato, Parere n. 351/2017, 13 febbraio, su Decreto MIT relativo alla programmazione triennale e biennale, cit

${ }^{32}$ En el mismo sentido se expresan los considerandos 2, 47, 96 y 121 de la Directiva 2014/24/UE y que suponen la confirmación de lo ya indicado en Comisión Europea, Libro Verde sobre la modernización de la política de contratación pública de la UE. Hacia un mercado europeo de la contratación pública más eficiente, Bruselas, 2011, pp. 4 y 5; ANAC, Determinazione n. 5/2013, 6 novembre: Linee guida su programmazione, progettazione ed esecuzione del contratto nei servizi e nelle forniture.
} 


\section{La planificación de los contratos públicos como posible fuente de transparencia administrativa. El caso italiano}

procedimientos de compra al dar lugar a una suerte de motivación-planificación inicial de los subsiguientes procedimientos de compra pública que permitirá potencialmente su contraste, interconexión e incluso control de legalidad respecto de las motivación-ejecución de los procedimientos ulteriores33, así como su la fiscalización de su ulterior desarrollo por cualquier ciudadano (Costanzo, 2000: 358; Garofoli y Ferrari, 2017: 585 y 586; Mattarella, 2016: 352). Hemos de destacar en este punto la cita llevada cabo por Matarella de Brandeis (1913) quien viene a señalar que: "se dice que la luz del sol es el mejor desinfectante y la luz eléctrica el policía más eficiente".

Ventajas de esta mayor transparencia producida por las programaciones de los procedimientos de contratación que se han tratado de incrementar en ambos casos sobre la base de los efectos potenciadores de las TIC mediante una completa digitalización de ambos tipos de anuncios dirigida a la generación de "información digital", dado que, tanto el programa bienal de bienes y servicios como el trienal de obras públicas y sus actualizaciones anuales, deben de ser publicados en el perfil del contratante, en la web del Ministerio de Infraestructuras y transportes y en el Observatorio de la contratación pública ${ }^{34}$-art. 21.7 del d.lgs. n. 50/2016-, con el formato de open data tal y como viene a determinar expresamente en relación a la programación trienal de obras públicas el art. 5.5 del decreto n. 14/2018, sin perjuicio de que también deba adoptarse dicho formato para las programaciones bienales por aplicación de la normativa general -art. 7 del d.lgs. 33/2013-.

Así las cosas, en el Ordenamiento Jurídico italiano podemos encontrar una experiencia legislativa cuyo seguimiento merece la pena llevar a cabo al constituir, potencialmente, una herramienta que puede ser verdaderamente útil a la hora de facilitar y potenciar el control difuso por parte de los ciudadanos en general y de los operadores económicos en particular de la actividad contractual de los entes adjudicadores, alumbrando una senda que en nuestro Ordenamiento Jurídico trata de abrir el art. 28.4 de la Ley 9/2017.

\footnotetext{
33 Sobre la relación inversamente proporcional entre el nivel de transparencia y la extensión de comportamientos conscientemente irregulares por parte de los responsables de los entes adjudicadores véase Comisión Europea, COM(2004) 841 de 29 de diciembre: Plan de acción para la aplicación del marco jurídico de la contratación pública electrónica, p. 9; COMISIÓN EUROPEA, COM(96) 583 final. Libro verde. La contratación pública en la Unión Europea: Reflexiones para el futuro, Bruselas, 1996, p. 27; Comisión Europea, COM(2003) 567, de 26 de septiembre: El papel de la administración electrónica en el futuro de Europa, punto b); Considerando 52 de la Directiva 2014/24/UE; Presidenza del Consiglio dei Ministri, Circolare n. 1/2010: Uso della Posta elettronica certificata nelle amministrazioni pubbliche.

34 Tal y como estableció el Comunicado de 26 de octubre de 2016 del Presidente de la ANAC: "mientras se procede a la adecuación definitiva de los sistemas informáticos de la Autoridad, las administraciones adjudicadoras deben proceder al cumplimiento de las obligaciones previstas en el art. 21, epígrafe 7 del código [de contratos públicos] mediante la publicación de las programaciones en su perfil del contratante y en el portal web del Ministero delle Infrastrutture e dei Trasporti".
} 


\section{Alfonso Sánchez García}

\section{BIBLIOGRAFÍA}

BRANDEIS, L., 1913, "What publicity can do", Harper's Weekly.

CAMINITI, D., 2017, "Art. 21. Programma degli acquisti e programmazione dei lavori pubblici", en GAROFOLI, R. y FERRARI, G. (coords.) Codice dei contratti pubblici. Tomo II, Molfetta, Neldiritto editore, pp. 351-376.

CARINGella, F.; y GiUstiniani, M., 2015, Manuale dei contratti pubblici, Roma, Dike. Giuridica Editrice.

CARINGella, F.; y PROTto, M., 2017, Il codice dei contratti pubblici dopo il correttivo. Commento organico al codice e alle linee guida ANAC alla luce del decreto corretivo del 19 aprile 2017, n. 56, Roma, Dike. Giuridica Editrice.

ClARICH, M., 2016, "Contratti Pubblici e concorrenza", en La nuova disciplina dei contratti pubblici. Tra esigenze di semplificazione, rilancio dell'economia e contrasto alla corruzione, Milano, Giuffrè Editore, pp. 263-303.

COMBA, M. E., 2014, "Variations in the scope of the new EU public procurement Directives of 2014: Efficiency in public spending and major role of the approximation of laws", en LICHĖRE, F., CARANTA, R. y TREUMER, S. (coords.) Modernising Public Procurement: The New Directive, Copenhagen, Djof Publishing, pp. 29-48.

Costanzo, P., 2000, "Internet (Diritto Pubblico) -voce-", en Digesto discipline pubblicistiche. Aggiornamento, Torino, Utet giuridica, pp. 347-371.

DugATO, M., 2015, "Organizzazione delle amministrazioni aggiudicatrici e contrasto alla corruzione nel settore degli appalti pubblici", Munvs, vol. 3, pp. 666-684.

DuGATO, M., 2017, "Terremoto, ricostruzione e regole degli appalti", Munvs, vol. 3, pp. 485-496.

GALLI, D.; GENTILE, D.; y GUALANDI, V. P. (coords.), 2015, "L'attività di programmazione dei contratti pubblici e la determina a contrarre", en Appalti pubblici, Milanofiori Assago, Wolters Kluwer, pp. 214-225.

GIANNINI, M. S., 1977, Diritto pubblico dell'economia, Bologna, Il Mulino.

MACHLUP, F., 1979, "Teorías de la empresa: Marginalista, Behaviorista, Gerencial", Revista Española de Economía, vol. IX.

MAtTARELlA, B. G., 2016, "Disciplina dei contratti pubblici e prevenzione della corruzione", en La nuova disciplina dei contratti pubblici. Tra esigenze di semplificazione, rilancio dell'economia e contrasto alla corruzione. Milano, Giuffrè Editore, pp. 337-353. 


\section{La planificación de los contratos públicos como posible fuente de transparencia administrativa. El caso italiano}

MELE, E., 2011, I contratti delle Pubbliche Amministrazioni, Milano, Giuffrè Editore.

Monchón, F., 1979, "Teorías alternativas de la empresa", Revista Española de Economía, vol. IX.

PONTI, B., 2005, "Organizzazione delle pubbliche amministrazioni. Rapporti fra Stato, regioni e autonomie locali", en CARLONI, E. (coord.) Codice dell'amministrazione digitale. Commento al D.Lgs. 7 marzo 2005, n. 82, Santarcangelo di Romagna, Maggioli Editore, pp. 129-162.

SCIANCALEPORE, V. D., 2017, "Pianificazione, programmazione e progettazione", en CORRADINO, M. y DAMIANI, S.S. (coords.) I nuovi appalti pubblici. Commento al d.lgs. 18 aprile 2016, n. 50, Varese, Giuffrè Editore, pp. 43-68.

ScocA, S. S., 2008, Evidenza pubblica e contratto: profili sostanziali e processuali, Milano, Giuffrè Editore.

TASSONE, A. R., 2016, "Il codice dei contratti pubblici e le autonomie", en SAITTA, F. (coord.) Appalti e contratti pubblici. Commentario sistematico, Wolters Kluwer; Milanofiori Assago, CEDAM, capitolo IV.

VAIANO, D., 2017, "Art. 29. Principi in materia di trasparenza", en GAROFOLI, R. y FERRARI, G. (coords.) Codice dei contratti pubblici. Tomo I, Molfetta, Neldiritto editore, pp. 583-594. 